\title{
Identification of models of nonlinear dynamic processes in mining on the basis of Volterra nuclei
}

\author{
Natalia Morkun ${ }^{1 *}$, Iryna Zavsiehdashnia ${ }^{1}$, Oleksandra Serdiuk ${ }^{1}$, and Iryna Kasatkina ${ }^{1}$ \\ ${ }^{1}$ Kryvyi Rih National University, Department of Automation, Computer Sciences and Technology, \\ 11 Matusevycha St., 50027 Kryvyi Rih, Ukraine
}

\begin{abstract}
Solving the problem of improving efficiency of technological processes of mineral concentration is one of the essential for providing sustainability of mining enterprises. Currently, special attention is paid to optimization of technological processes in concentration of useful minerals. This approach calls for availability of high-quality data on the process, formation of corresponding databases and their subsequent processing to build adequate and efficient mathematical models of processes and systems. In order to improve quality of mathematical description of forming fractional characteristics of ore through applying technological aggregates in concentration, the authors suggest using power Volterra series that provide characteristics of a controlled object (its condition) as a sequence of multidimensional weight functions invariant to the type of an input signal - Volterra nuclei. Application of Volterra structures enables decreasing the modelling error to 0.039 under the root-mean-square error of 0.0594 .
\end{abstract}

\section{Introduction}

Metallurgy is one of the most essential Ukrainian industries. Analysis of current conditions and trends of Ukraine's mining and metallurgical industry reveals considerable problems caused by severe competitiveness and gradual deterioration of quality of processed ores. Methods and algorithms of controlling technological processes in mineral concentration are unable to reduce power consumption, improve quality and productivity, minimize losses, etc. Therefore, leading mining countries assume that creation of new highly efficient methods and algorithms of automated control over technological processes in ore concentration through improving data support and control methods, particularly in characteristics of technological flows of a concentration plant is a priority.

The issue of automated control over multifactor energy-intensive technological processes when there are variations of processed raw material characteristics caused by managerial-technical and random factors is one of the most complicated tasks calling for application of specific methods. Formation of optimal control over concentration processes as interrelated space-distributed blocks is one of such processes.

\footnotetext{
* Corresponding author: nmorkun $@$ a,knu.edu.ua
} 
As in case of systems with concentrated parameters, basic forms to describe distributed objects include presenting them by differential equations in partial derivatives, transmission functions, time characteristics, and frequency characteristics [1 - 4].

In [4-6] there are two approaches to forming a time-space model of the distributed system. The former approach implies determining the system's reaction to the input signal presented as a combination of delta functions in space and time domains $[7,8]$ :

$$
\omega\left(x_{1}, t\right)=\delta\left(x_{1}-\rho_{0}\right) \delta\left(t-\tau_{0}\right),
$$

where $x_{1} \in D_{1}, \rho_{0} \in D_{2} ; x_{1}, \rho_{0}$ are assigned space points $D_{1}, D_{2} ; t, \tau$ are time independent variables.

The object's reaction to the input impact $\omega(x, t)$ is presented as the Grin function $G(x, t, \rho, \tau)$ or an impulse transitional function. The latter approach involves determining the object's reactions to its own vector-functions of the object operator. Under such circumstances, the distributed object is an infinite totality of independent conventionally concentrated contours, transitional functions of each of them are presented as a ratio of analytical integral functions.

[9-11] analyzes peculiarities of basic forms of describing distributed objects. It is indicated that distributed systems are noted for space-related components in input and output signals. That is why, in distributed systems, time-related inputs should be complimented with their spacious form. In case of a linear distributed object, dependency of the output function $Q(x, t)$ on the input impact $\omega(\rho, \tau)$ can be presented as follows:

$$
Q(x, t)=\int_{t_{0}}^{t} \int_{D_{1}} G(x, t, \rho, \tau) \omega(\rho, \tau) d \rho d \tau,
$$

where $G(x, t, \rho, \tau)$ is an impulse transitional function (the Grin function). The integral ratio for the output function is determined in compliance with the following expression:

$$
Q(x, t)=G(x, t, \rho, \tau) \bar{\otimes} \omega(\rho, \tau),
$$

where $\bar{\otimes}$ is an operator to integrate two functions related to this symbol by the parameters $\rho \in D_{1}$ and $\tau$. The time distributed block is described by the following ratio in Laplace transform terms:

$$
\bar{Q}(x, s)=\int_{D_{1}} W(x, \rho, s) \bar{\omega}(\rho, s) d \rho,
$$

where $Q(x, s), \omega(\rho, s)$ is Laplace transform of the functions $Q(x, t)$ and $\omega(\xi, \tau)$ respectively.

Transition to mathematical modeling of distributed systems by using neural networks is considered in $[11,12]$. The authors think that this approach is applicable if the function describing the controlled object is characterized by the following properties. It is a composition of simple elements; its structure is defined by selecting parameters from the finite set and selection procedures are resistant to disturbances of initial data and calculating errors. It is indicated that analytical solution provides for a small number of problems, while current approximating methods of solution either allow obtaining only pointwise approximation similar to network methods or advance specific demands to selecting approximation functions. The advantage of the neural-network approach is the opportunity to obtain a solution in the form of a function, thus meeting the requirements of smoothness. The most important advantage of neural-network model is its resistance to data errors inaccuracies in setting equation coefficients, boundary and initial conditions, disturbances of the boundary, calculating errors, etc. 
$[12,13]$ suggest applying neural networks to solving differential equations with partial derivatives. Also, $[10,13,14]$ investigates into the neural-network variant of the splinecollocation method for numerical solution of nonlinear equations of mathematical physics. At the same time, it is indicated in $[15,16]$ that there are some disadvantages in combining neural-network approximation of solutions of the boundary value problem with the collocation method under invariable basic functions.

To increase efficiency of methods of identifying nonlinear dynamic systems, $[2,5,17]$ suggests Volterra models. At the same time, the robust method for identifying nonlinear dynamic systems based on Volterra models in the time domain has been developed. It provides for solving integral first-kind Volterra equations through applying the method of standardizing incorrect problems developed by A.M. Tikhonov that enables increased accuracy of identification [18].

Thus, considering spacious distribution of the technological line in concentration as a controlled object, among available approaches to modelling, the most promising ones include those providing formation of a model as a system with distributed parameters.

The suggested model of the control system is based on presenting a technological concentration line as a structure with concentrated input impacts and an output distributed along the entire technological line - the function of distributing the Fe content in size classes of ore particles. A set of ultrasonic and radiometric measurements enables efficient assessment of distributing the useful component in distinguished iron ore classes $[6,8,14]$.

\section{The mathematical model and the mechanism of applying Volterra nuclei}

The problem of identifying the second-kind Volterra model can be solved by forming equations to assess model parameters envisaging the minimal root-mean-square error of forecasting and simultaneously admitting that the input sequence $\{\psi(k)\}$ is stationary [19, 20]. The advantage of this approach implies appearance of simple equations assessment with certain correlations that can be assessed by available data.

Equations assessing parameters are synthesized by using the expression of the meansquare forecasting error:

$$
M S E=E\left\{\left(\beta^{d}(k)-\beta_{0}^{d}-\sum_{i=0}^{M} \alpha_{1}(i) \psi(k-i)-\sum_{i=0}^{M} \sum_{j=0}^{M} \alpha_{2}(i, j) \psi(k-i) \psi(k-j)\right)^{2}\right\} .
$$

Through differentiating equation (1) according to the constant parameter $\beta_{0}^{d}$, we obtain the following ratio between this parameter and the second-order model coefficients $a_{2}(i, j)$ $[20,21]$ :

$$
\beta_{0}=\bar{y}-\sum_{i=0}^{M} \sum_{j=0}^{M} a_{2}(i, j) R_{\psi \psi}(i-j) .
$$

Similarly, by differentiating equation (5) according to the mean-square parameter $a_{2}(p, q)$, we obtain a system of linear equations:

$$
\sum_{i=0}^{M} \sum_{j=0}^{M} a_{2}(i, j) E\{\psi(k-i)(k-j) \psi(k-p) \psi(k-q)\}=t_{\psi \beta}(p, q)-\beta_{0} R_{\psi \psi}(p-q) .
$$

Substituting equations (6) into (7) involves a fixed term $y_{0}$ that under $p, q=0,1, \ldots, M$ results into the following system of nonlinear equations for $a_{2}(i, j)[20,22]$ : 


$$
\sum_{\alpha 0}^{M} \sum_{j=0}^{M} D(i, j, p, q) a_{2}(i, j)=t_{\psi w}(p, q),
$$

where $t_{\psi w}(p, q)$ is a reciprocal bicorrelation between the initial sequence $\{\psi\}$ and the initial deviation $w(k)=\beta(k)-\bar{\beta} ; D(i, j, p, q)$ determined by the formula:

$$
D(i, j, p, q)=E\{\psi(k-i) \psi(k-j) \psi(k-p) \psi(k-q)\}-R_{\psi \psi}(p-q) .
$$

In equation (8), the parameter $D$ can be expressed in a simpler way in case of the known distribution of the input sequence. In case of the Gaussian distribution considered in [23], $D$ is set by the formula:

$$
D(i, j, p, q)=R_{\psi \psi}(i-p) R_{\psi \psi}(j-q)+R_{\psi \psi}(i-q) R_{\psi \psi}(j-p) .
$$

Considering that $a_{2}(i, j)=a_{2}(j, i)$ and $t_{\psi w}(p, q)=t_{\psi w}(q, p)$, equation (8) is written as:

$$
2 \sum_{i=0}^{M} \sum_{j=0}^{M} R_{\psi \psi}(i-p) R_{\psi \psi}(j-q) a_{2}(i, j)=t_{\psi w}(p, q) .
$$

According to the definition of the matrix multiplication operation, the equation can be rewritten in the following way:

$$
2 R_{\psi \psi} B R_{\psi \psi}=T_{\psi w}
$$

where $B$ is the matrix of $(M 1) \times(M+1)$ the $\mathrm{i}$, $\mathrm{j}$ elements of which are $t_{\psi w}(i, j)$. Considering that the input autocorrelation matrix $R_{\psi / \psi}$ is reciprocal, equation (8) has the following analytical solution for the problem of identifying the second-order Volterra model:

$$
B=\frac{1}{2} R_{\psi \psi}^{-1} T_{\psi \psi} R_{\psi \psi}^{-1}
$$

Identification of the third-order Volterra model is more complicated and, as a result, it is used less often in practice $[24,25]$. As in case of the second-order model, to identify thirdorder models, there are used results of factorizing the higher-order moments to synthesize equations and determine model parameters on the basis of reciprocal three-correlation of input and output signals. The structure of these results is more complicated than for the second-order model as conditions for solving the second-order problem do not solve the third-order equation to the full extend. It should be noted that the symmetric component of the system response does not result from the linear part of the Volterra model only, it including parameters of the third-order model as well.

The above issues are studied in $[14,26]$ in detail. Simplification of identification is performed by reducing the input sequence to the zero average. Yet, [21] does not envisage symmetrical distribution of the input sequence and, therefore, moments of odd orders are nonzero. Similarly to the way of the second-order solution including the fourth moments up to excess, the third-order solution includes sixth moments. [23] suggests the expression for mathematical expectation in a general form $\operatorname{Ex}\left\{\left(k_{1}\right) \ldots x\left(k_{n}\right)\right\}$ when $n=2,3,4,5$ and 6 . For instance, when $n=6$, we have: 


$$
\operatorname{Ex}\left\{\left(k_{1}\right) \ldots x\left(k_{n}\right)\right\}=\left\{\begin{array} { l } 
{ \mu _ { 6 } } \\
{ \mu _ { 4 } \mu _ { 2 } } \\
{ \mu _ { 3 } ^ { 2 } } \\
{ \mu _ { 2 } ^ { 3 } } \\
{ 0 }
\end{array} \quad \ldots \left\{\begin{array}{l}
k_{1}=\ldots=k_{6} \\
k_{1}=k_{2}<k_{3}=k_{4}=k_{5}=k_{6} \\
k_{1}=k_{2}=k_{3}<k_{4}=k_{5}=k_{6} \\
k_{1}=k_{2}<k_{3}=k_{4}<k_{5}=k_{6} \\
\text { otherwise }
\end{array} .\right.\right.
$$

Considering these results, as in case of the second order, one can obtain clear solutions of the assessment problem by the least-square method that leads finally to comparatively accurate results of the Volterra model parameters. The simplest of these equations is the one for $a_{3}\left(i_{1}, i_{2}, i_{3}\right)$ when $i_{1}<i_{2}<i_{3}$ :

$$
\hat{a}_{3}\left(i_{1}, i_{2}, i_{3}\right)=\frac{E\left\{\beta(k) \psi\left(k-i_{1}\right) \psi\left(k-i_{2}\right) \psi\left(k-i_{3}\right)\right\}}{\mu_{2}^{3}} .
$$

The equation for assessing other model parameters are closely connected with other assessments of parameters and therefore they should be solved in a certain sequence. Nondiagonal assessments of quadratic parameters $\hat{a}_{2}\left(i_{1}, i_{2}\right)$ for $i_{1}<i_{2}$ are set by the expression:

$$
\hat{a}_{2}\left(i_{1}, i_{2}\right)=\mu_{2}^{-2} \alpha-\mu_{2}^{-1} \mu_{3}\left[\hat{a}_{3}\left(i_{1}, i_{1}, i_{2}\right)+\hat{a}_{3}\left(i_{1}, i_{2}, i_{2}\right)\right]
$$

Diagonal parameters $a_{2}\left(i_{1}, i_{2}\right)$ and $a_{3}\left(i_{1}, i_{2}, i_{3}\right)$ should be calculated only after determining all nondiagonal parameters of the second and third order. Linear parameters should be calculated last of all as formulae for their calculations include diagonal parameters.

\section{Results and discussion}

To investigate into efficiency of the developed model, a hybrid Hammerstein model has been used as a basic one, it consisting of two elements - the stationary nonlinear block (" $\psi-v$ " channel) described by the fuzzy model and the linear dynamic block (" $v-\beta$ " channel). According to this structure, at the output of the stationary nonlinear block based on the fuzzy knowledgebase, we obtain data not on input variables $\beta^{d}(k)$, but transformed input ones $v(k)$. The output of the fuzzy model is calculated as the weight-average number of fuzzy rule consequences:

$$
v_{h}=\frac{\sum_{i_{1}=1}^{M_{1}} \cdots \sum_{i_{n}=1}^{M_{n}} \vartheta_{i_{1}, \ldots, i_{n}}(\psi) d_{h, i_{1} \ldots i_{n}}}{\sum_{i_{1}=1}^{M_{1}} \cdots \sum_{i_{n}=1}^{M_{n}} \vartheta_{i_{1}, \ldots, i_{n}}(\psi)},
$$

where $M_{j}$ is the number of fuzzy sets in the $j$-th input domain; the execution rate is $\vartheta_{i_{1}, \ldots, i_{n_{\psi}}}(\psi)=\prod_{j=1}^{n_{u}} A_{j, \ldots, i_{j}}\left(\psi_{j}\right)$.

To approximate the dynamic block in this model, difference equations are used. The hybrid Hammerstein model - block-oriented model, which represents a non-linear system in the form of various combinations of inertial links and non-linear inertial mathematical elements - at the $(k+1)$-th step looks like [20,26]: 


$$
\beta^{d}(k+1)=\sum_{i=1}^{n_{y}} A_{i} \beta^{d}(k-i+1)+\sum_{i=1}^{n_{u}} B_{i} \sum_{i_{1}=1}^{M_{1}} \ldots \sum_{i_{n}=1}^{M_{n}} \vartheta_{i_{1} \ldots i_{n}}\left(\psi\left(k-i-n_{d}+1\right)\right) d_{h, i_{1} \ldots i_{n}},
$$

where $\beta^{d}(k-i+1)$ and $\psi\left(k-i-n_{d}+1\right)$ are corresponding outputs and inputs at previous steps; $n_{\beta}$ and $n_{\psi}$ are maximum delays for outputs and inputs; $n_{d}$ is a one-time discrete delay (transport); $A_{i}$ and $B_{i}$ are matrices of $m \times m$ and $m \times n$ respectively that characterize the linear dynamic block; $d_{h, j}$ is the parameter characterizing the nonlinear (fuzzy) block.

Models of nonlinear dynamic objects in concentration are identified according to the above procedure on the basis of testing results of the technological concentration line.

At the same time the following parameters are used: efficiency of ore mining; water consumption; yield of concentrate, industrial products and tailings; yield of control sizes; content of $F e_{t o t}, F_{m}$ associated with magnetite $\left(F e_{m}\right)$, hematite $\left(F e_{g e m}\right)$ and siderite $\left(F e_{s i d}\right)$; power consumption of the technological line and basic technological aggregates.

Fig. 1 shows changes of the error (MSE) during identification without noises in the output signal channel. The graphs of MSE changes in identifying under additive noises of various capacity in the output signal channel are presented in Figs. 2 and 3. The obtained graphs of errors of the basic model and the Volterra model are presented in Figs. 4 and 5.

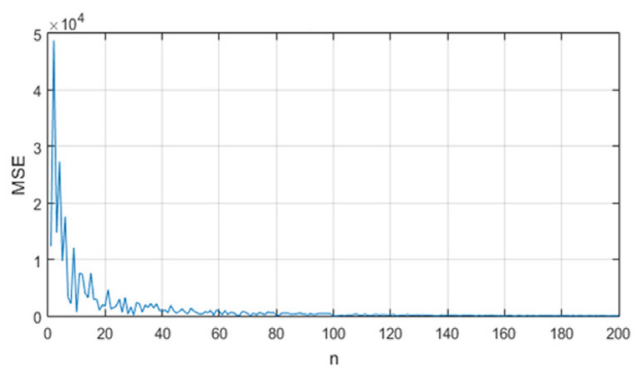

Fig. 1. The error (MSE) without noises at the object output.

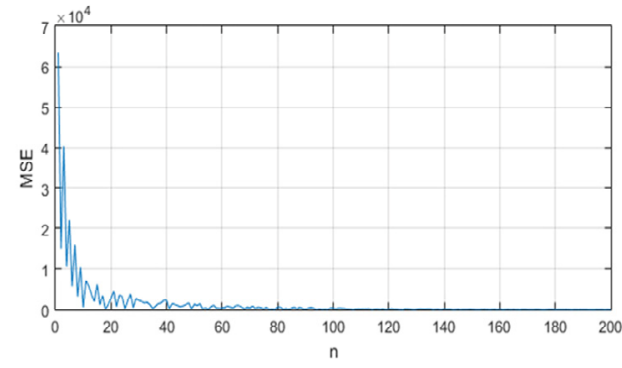

Fig. 3. The error (MSE) under noises at the object output $\sigma=00.1$.

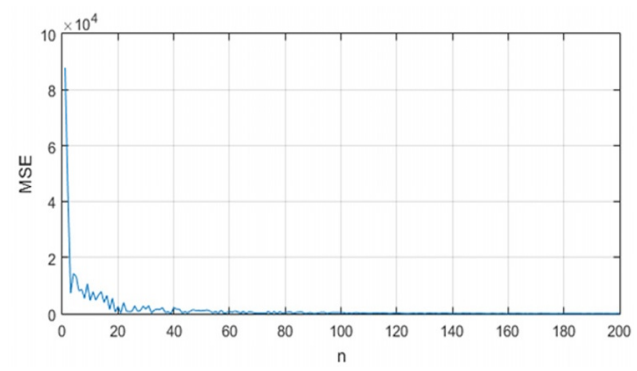

Fig. 2. The error (MSE) under noises at the object output $\sigma=0.1$.

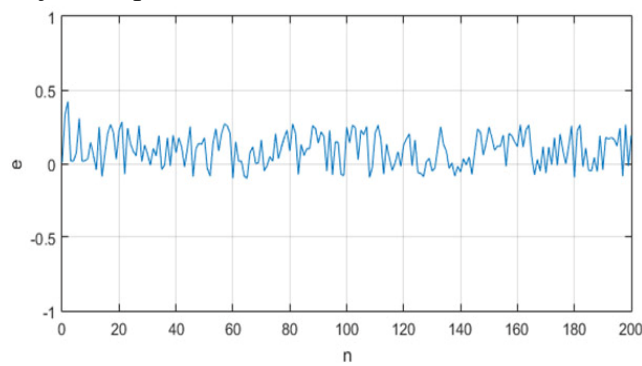

Fig. 4. Errors of the basic model.

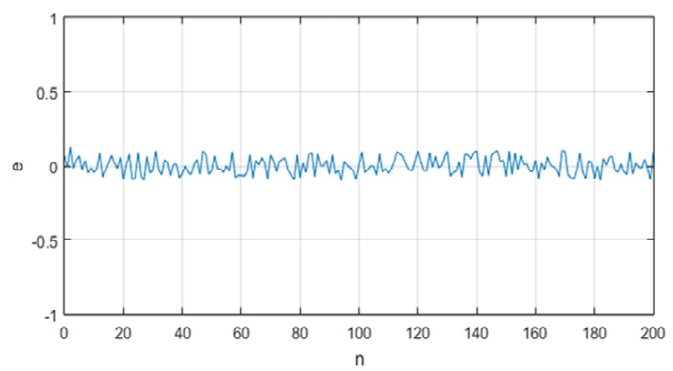

Fig. 5. Errors of the Volterra model. 
Table 1 presents the modelling results. The hybrid Hammerstein model suggested in [25] is used as a basic one with the nonlinear block presented by the fuzzy model of the Takagi-Sugeno type and connected with the dynamic block based on autoregressive equations.

Table 1. Analysis of ore concentration models.

\begin{tabular}{|c|c|c|c|c|}
\hline No. & Model & Mean error $\varepsilon_{m}$ & Dispersion $D(\varepsilon)$ & MSW $\sigma(\varepsilon)$ \\
\hline 1 & Basic & 0.0915 & 0.0132 & 0.1151 \\
\hline 2 & Volterra & 0.039 & 0.035 & 0.0594 \\
\hline
\end{tabular}

Thus, it makes sense to apply Volterra structures to improving quality of mathematical description while forming fractional characteristics of ore by technological aggregates of concentration, this enabling the modeling error to fall by a factor of 0.039 with the rootmean-square deviation of 0.0594 .

\section{Conclusions}

The proposed control system model is based on the representation of the ore-benefication process line as a structure with concentrated input influences and the output distributed over the entire production line - a function of the distribution of iron level over the particle size classes of ore particles.

To form time-space models of nonlinear dynamic objects in concentration, it is reasonable to refer to them as operators transforming vectors of input variables into those of output parameters.

Application of the Volterra model enables decreasing the modelling error up to 5.94\%, which serves a confirmation for viability of the given approach to identify processes of iron ore concentration. Yet, there is necessity of a great number of coefficients required for its implementation.

Application of the suggested method of identifying nonlinear dynamic objects in concentration on the basis of the time-space Volterra model will enhance quality of automated control over parameters of iron ore concentration under conditions of nonstationarity and changes of static and dynamic parameters of an object.

The search for ways to eliminate considerable disadvantages of the Volterra model is planned to be the next stage of our further research.

Numerical data are provided in the research report "Identification of nonlinear dynamic technological objects of concentration based on nucleus operators" (State registration No. 0116U001518).

\section{References}

1. Akaike, H. (1974). A new look at the statistical model identification. IEEE Transactions on Automatic Control, 19(6), 716-723. https://doi.org/10.1109/tac.1974.1100705

2. Boyd, S., \& Chua, L. (1985). Fading memory and the problem of approximating nonlinear operators with Volterra series. IEEE Transactions on Circuits and Systems, 32(11), 1150-1161. https://doi.org/10.1109/tcs.1985.1085649

3. Golik, V., Komashchenko, V., \& Morkun, V. (2015) Feasibility of using the mill tailings for preparation of self-hardening mixtures. Metallurgical and Mining Industry, 7(3), 38-41.

4. Morkun, V., \& Tron, V. (2014) Ecological and economic optimization of iron ore processing automated control. Metallurgical and Mining Industry, 6(5), 8-10. 
5. Ching-Hsiang Tseng, \& Powers, E.J. (1995). Identification of cubic systems using higher order moments of i.i.d. signals. IEEE Transactions on Signal Processing, 43(7), 1733-1735. https://doi.org/10.1109/78.398739

6. Morkun, V., Morkun, N., \& Pikilnyak, A. (2015). The study of volume ultrasonic waves propagation in the gas-containing iron ore pulp. Ultrasonics, (56), 340-343. https://doi.org/10.1016/j.ultras.2014.08.022

7. Butkovskij, A.G. (1965). Teoriya optimal'nogo upravleniya sistemami s raspredelennymi parametrami. Moskva: Nauka.

8. Morkun, V., Morkun, N. \& Pikilnyak, A. (2014) Ultrasonic facilities for the ground materials characteristics control. Metallurgical and Mining Industry, (2), 31-35.

9. Grigor'ev, V.V., \& Bystrov, S.V., \& Pershin, I.M. (2010) Sintez raspredelennykh regulyatorov. Sankt-Peterburg: SPbGU ITMO.

10. Golik, V., Komashchenko, V., Morkun, V., \& Burdzieva, O. (2015) Metal deposits combined development experience. Metallurgical and Mining Industry, (6), 591-594.

11. Pershin, I.M. (2013). Sistemy s raspredelennymi parametrami. Pyatigorsk: SKFU.

12. Vasil'ev A.N. (2007). Matematicheskoe modelirovanie raspredelennykh sistem s pomoshchyu neyronnykh setey. Matematicheskoe Modelirovanie, 12(19), 32-42.

13. Galushkin, A.I (2002). Neyromatematika. Moskva.

14. Morkun, V., Morkun, N., \& Pikilnyak, A. (2015). Adaptive control system of ore beneficiation process based on Kaczmarz projection algorithm. Metallurgical and Mining Industry, (2), 35-38.

15. Qi Chenkun. (2009). Modeling of nonlinear distributed parameter system for industrial thermal processes. PhD Thesis. Hong Kong, China.

16. Pavlenko V.D. (2016). Methods and tools for identification nonlinear dynamical systems with using Volterra models. PhD Thesis. Odesa, Ukraine.

17. Morkun, V. \& Tron, V. (2014) Automation of iron ore raw materials beneficiation with the operational recognition of its varieties in process streams. Metallurgical and Mining Industry, (6), 4-7.

18. Porkuyan, O.V. (2009). Control of nonlinear dynamic objects of concentrating productions on the basis of Hammerstein hybrid models. PhD Thesis. Kryvyi Rih, Ukraine.

19. Golik, V., Komashchenko, V., Morkun, V., \& Zaalishvili, V. (2015). Enhancement of lost ore production efficiency by usage of canopies. Metallurgical and Mining Industry, (4), 325-329.

20. Doyle, F.J. III, Pearson, R.K., \& Ogunnaike, B.A. (2002). Identification and Control Using Volterra Models. London.

21. Taiho Koh, \& Powers, E. (1985). Second-order Volterra filtering and its application to nonlinear system identification. IEEE Transactions on Acoustics, Speech, and Signal Processing, 33(6), 1445-1455. https://doi.org/10.1109/tassp.1985.1164730

22. Golik, V., Komashchenko, V., Morkun, V., \& Irina, G. (2015). Improving the effectiveness of explosive breaking on the bade of new methods of borehole charges initiation in quarries. Metallurgical and Mining Industry, 7(7), 383-387.

23. Li, H.-X., \& Qi, C. (2009). Incremental Modeling of Nonlinear Distributed Parameter Processes via Spatiotemporal Kernel Series Expansion. Industrial \& Engineering Chemistry Research, 48(6), 3052-3058. https://doi.org/10.1021/ie801184a

24. Morkun, V., \& Tcvirkun, S. (2014). Investigation of methods of fuzzy clustering for determining ore types. Metallurgical and Mining Industry, (5), 11-14.

25. Zheng, Q., \& Zafiriou, E. (2004). Volterra-Laguerre Models for Nonlinear Process Identification with Application to a Fluid Catalytic Cracking Unit. Industrial \& Engineering Chemistry Research, 43(2), 340-348. https://doi.org/10.1021/ie021064g

26. Abhishek S. Soni. (2006). Control-Relevant System Identifiction Using Nonlinear Volterra and Volterra-Laguerre Models. PhD thesis. Pittsburgh, USA. 